\title{
WHAT IS THIS THAT STANDS BEFORE ME? MÄNNERBILdER IM HEAVy METAL
}

\section{Dietmar Elflein}

»What is this that stands before me? « Mit dieser Frage beginnt das Stück »Black Sabbath«, welches das gleichnamige Debüt der ebenfalls gleichnamigen Band eröffnet. Diese 1970 erschienene LP wird in der Heavy MetalGeschichtsschreibung im Allgemeinen als eine der ersten, wenn nicht die erste Heavy Metal-Schallplatte betrachtet (vgl. Christie 2003: 4; Weinstein 1991: 14). Mit dieser Frage beginnt somit auch der lyrische Kosmos des Heavy Metal. Adressat der Zeile ist eine dunkle Macht, vor der der Protagonist des Songs flüchten muss. Er muss jedoch die Sinnlosigkeit seines Tuns einsehen und ruft Gott um Hilfe an: »Oh no, no, please, God, help me!«

Wenn man - zugegebenermaßen etwas willkürlich - annimmt, dass dieser lyrische Protagonist männlichen Geschlechts ist, ${ }^{1}$ so erscheint am Beginn der Heavy Metal-Geschichtsschreibung ein Männerbild, das unter anderem Elemente der Verletzlichkeit und der Angst enthält. Hier spricht definitiv kein Held.

Die Frage »What is this that stands before me?« dient deshalb als Anlass zu fragen, aus welchen Zutaten Männerbilder im Heavy Metal konstruiert sind. Was hat es auf sich mit dem Klischee des langhaarigen, tätowierten Machos, der seine Gitarre als Phallusverlängerung nutzt? Stellt sich das Männerbild im Heavy Metal vielschichtiger dar, als es auf den ersten Blick scheint?

\section{I - Die zentrale These und eine Begriffsklärung}

Die zentrale These meines Aufsatzes ist, dass ein mit Pierre Bourdieu (2005) und David Savran (1998) für die westliche Welt als hegemonial behaupteter Habitus des weißen Mannes in der Kulturwelt Heavy Metal sowohl Zustim-

1 Der Protagonist bleibt geschlechtslos, Textdichter und Interpret sind jedoch männlichen Geschlechts. 
mung als auch Ablehnung erfährt. Gleichwohl imaginiert die Kulturwelt ihre Ablehnung des hegemonialen Habitus als überwiegend und glaubt sich gerüstet, diesen in transgressiven Akten sozusagen spielerisch und gefahrlos ausleben zu können. Die dunkle Macht, vor der Ozzy Osbournes Protagonist im Aufsatztitel flüchtet, wäre damit metaphorisch gesprochen die momentan hegemoniale Männlichkeit, deren Anziehungskraft trotzdem unbestritten bleibt.

Die hier in Frage stehenden Männerbilder dienen mehrheitlich als Imageträger im Rahmen des geschäftlichen Interesses der Musikindustrie. Grundlage meiner Betrachtungen sind Pressefotos und Musikvideos bzw. offiziell veröffentlichte Konzertmitschnitte. Aufgrund des mehr oder weniger inszenierten Charakters dieser Bilder ist diskutierbar, inwieweit das Konzept des Habitus auf derartige Images gewinnbringend angewendet werden kann. Christofer Jost schlägt den Brechtschen Gestus-Begriff als mögliche Alternative vor, sieht aber gleichzeitig die Verwurzelung des Begriffs im epischen Theater als ein möglicherweise zu problematisierendes Element an. ${ }^{2}$ Unter gestischem Bereich versteht Brecht (1964, Bd. 7: 48) »den Bereich der Haltungen, welche die Figuren zueinander einnehmen. [...] Körperhaltung, Tonfall und Gesichtsausdruck sind von einem gesellschaftlichen Gestus bestimmt.« »Ein Gestus zeichnet die Beziehungen der Menschen zueinander« (ebd., Bd. 6: 225). Nach Marc Silbermann (2003) soll er als kalkulierter Effekt die Gleichsetzung von Haltung und Ausdruck verhindern und damit auch die für das bürgerliche Subjekt grundlegende Unterscheidung von übergesellschaftlichem Individuum und sozialisierender Instanz, also Gesellschaft, konterkarieren.

Für Bourdieu stellt der sozialisierte Körper ebenfalls nicht das Gegenteil von Gesellschaft dar, sondern eine ihrer Existenzformen. Individuen sind bedingt durch ihre körperliche Existenz immer schon Mitglied von Gesellschaft. Gleichzeitig verkörpert sich die Gesellschaft in den Individuen, im Habitus als das Körper gewordene Soziale. Eine vorsoziale Subjektivität, ein Individuum, das erst vermittelt durch einen Sozialisationsprozess Teil von Gesellschaft wird, existiert für Bourdieu nicht (vgl. Engler 2008: 252f.).

Ähnlichkeiten von Gestus und Habitus finden sich damit in der Negierung der Innen-Außen-Spaltung des bürgerlichen Subjekts. Auch im Brechtschen Gestus verschwindet die Innerlichkeit als das Individuum konstituierender Raum - allerdings aufgrund seiner Begrenzung auf die Inszenierung in durchaus radikalerer Form als im Bourdieuschen Habitus. Brecht negiert die bürgerliche Individualität, Bourdieu relativiert sie. Josef Früchtl und Jörg

2 Diskussionsbeitrag im Anschluss an den Vortrag auf der Tagung des ASPM vom 25.-27.9.2009 in Halle. 
Zimmermann (2001: 13) formulieren bezüglich der Anwendung des - für den Gestus unabdingbaren - Inszenierungskonzeptes außerhalb der Theaterrealität zudem einige bedeutsame Einschränkungen und kommen zu dem Schluss, dass es der Abstützung durch andere Konzepte bedarf. So lässt sich mit dem Konzept des Habitus besser erklären »warum das Handeln von Personen weder wie ein auswendig gelerntes Buch und umgesetztes Skript noch wie eine Serie völlig freier Entscheidungen erscheint « (ebd.). Noch weiter gehend sieht Herbert Willems (2009: 21) Habitus als Schlüsselbegriff »von Medientheatralität und von der Bedingtheit von Medientheatralität «:

»Damit geht es auch um zentrale Seiten, Formen und Kontexte von Theatralität bzw. Medientheatralität: Stile (Verhaltensstile, Lebensstile), Images, Skripts, Rituale (Ritualisierungen), Strategien, Urteilsfähigkeiten und Mentalitäten sind als mehr oder weniger habitusbedingt oder/und habituserzeugt $\mathrm{zu}$ verstehen « (ebd.).

In diesem Sinne findet im Folgenden der Habitus-Begriff Verwendung, der sich unter anderem im Gestus verbildlichen kann.

\section{II - Die männliche Herrschaft oder Gender, Sex und Heavy Metal}

Der französische Soziologe Pierre Bourdieu (2005: 44f.) beschreibt in seinem Buch Die männliche Herrschaft eben jene als das Beispiel der symbolischen Gewalt par excellence. Seine Kernaussage ist, dass die sozial konstruierten Unterschiede zwischen den Geschlechtern ins Körperliche eingegangen sind und dadurch die männliche Herrschaft natürlich und unvermeidlich erscheinen lassen. Oder anders gesagt: Die männliche Herrschaft bezieht ihre Kraft und Langlebigkeit daraus, dass sie ein Herrschaftsverhältnis legitimiert, indem sie es einer biologischen Natur einprägt, die selbst eine naturalisierte gesellschaftliche Konstruktion ist.

Geschlecht wird damit als eine Dimension des Sozialen verstanden, als eine Dimension der Hervorbringung sozialer Wirklichkeit durch die Ein- und Aufteilung der sozialen Welt, wie sie von Akteuren und Akteurinnen vorgenommen wird. Als in den Habitus eingelagertes Klassifikationsschema, das von den Akteuren und Akteurinnen nicht oder nicht zwangsläufig erkannt wird, realisiert sich die Herrschaftsausübung in der symbolischen Gewalt, die in die alltäglichen Beziehungen ganz selbstverständlich eingeht. Dabei nimmt, »der Beherrschte den Herrschenden mittels Kategorien wahr, die 
von der Herrschaftsbeziehung hervorgebracht wurden und damit im Interesse des Herrschenden liegen« (Bourdieu 1998: 197).

»British Heavy Metal is neither misogynistic nor an expression of machismo, for the most part women are of no concern, there is no binary of male / female [...] that is, British Heavy Metal is not masculinist« (Weinstein 2009: 18).

Thesen zu Gender und Heavy Metal, wie die eben zitierte, verlieren auf dem Hintergrund von Bourdieus Überlegungen an Überzeugungskraft. Die Existenz eines männlichen Habitus ist auch in Männerbunden, für die Frauen > of no concern sind, als gegeben anzunehmen. Inwieweit die von Deena Weinstein ebenfalls in Abrede gestellte Frauenfeindlichkeit des britischen Heavy Metal im Heavy Metal eine größere oder auch kleinere Rolle spielt als in anderen Genres der Rockmusik, bleibt dagegen offen.

Darüber hinaus ist ein männlicher Habitus nicht an das biologische Geschlecht (Sex) gebunden. Beispielsweise beruht Extreme Metal-Gesang auf als aggressiv konnotierten Vokaltechniken, die vom einfachen Schreien über tiefes Grunzen bis zum stimmlosen Keifen reichen. Eine Podiumsdiskussion mit vier Heavy Metal-Sängerinnen auf dem Heavy Metal and Gender Kongress $^{3}$ im Oktober 2009 in Köln zeigte, dass die Beherrschung derartiger Stimmlagen durch Frauen häufig Reaktionen wie »Die singt ja wie ein Mann« provoziert. Zusätzlich zu diesen Gesangstechniken, die, wenn sie von Frauen beherrscht werden, allein schon traditionelle Genderstereotypen angreifen, verkörpern Sängerinnen wie Angela Gossow von Arch Enemy, Sabina Claasen von Holy Moses oder Britta Görtz von Cripper auf der Bühne auch eine Aggressivität und eine Sicherheit im Auftreten, die notwendig erscheint, um in die männliche Domäne des Extreme Metal-Gesangs einzubrechen. Im Interview für die Dokumentation Metal: A Headbanger's Journey (Dunn/McFayden 2006) spricht Angela Gossow von der Stärke und dem Selbstvertrauen, das sie auf der Bühne fühlt: »l am tough on stage, you know. I feel very strong on stage and I want to give that to the people in front of that stage. « Die begleitenden Konzertausschnitte zeigen sie mit weit ausholenden Armbewegungen mit geballter Faust und beim >Headbangen . Am Ende des Konzertausschnitts schlägt sie sich im Rhythmus des Breakdown mit der Faust auf den Brustkorb.

Damit wird die Trennung von Gender und Sex nochmals explizit deutlich. Männlichkeit und Weiblichkeit sind als Teile des jeweiligen Habitus sozial verkörpert. Bourdieus Ansatz unterstreicht in diesem Zusammenhang

3 Ausschnitte aus der Podiumsdiskussion sind auf der als Beilage zur Metal Hammer-Ausgabe vom Januar 2010 erschienenen DVD Maximum Metal 147 (V.A. 2009) veröffentlicht. 
sowohl die Widerstände, die Frauen in der Einnahme einer derartigen Position entgegenstehen (»Die singt ja wie ein Mann«), als auch die potentielle Veränderlichkeit des Feldes, die über die Existenz derartiger Sängerinnen bezeugt wird.

Im umgekehrten Sinne lassen sich auch die vielen Geschichten des Verschweigens und im Ergebnis des Vergessenwerdens von Musikerinnen in der Musikgeschichte mit Bourdieus Ansatz erklären. So zeigen Susan Fast (2009) und Gillian G. Gaar (1994) an diversen Beispielen den Mantel des Vergessens, der gerade über Gitarristinnen gerne gelegt wird, da E-Gitarre-Spielen laut Mavis Bayton (1997) männlich konnotiert ist.

Im Sinne von R.W. Connell (1995) existieren damit unterschiedliche Männlichkeiten, die nicht zwangsläufig mit dem biologischen Geschlecht verbunden sind, aber als Teil des Patriarchats, als naturalisierte, dem biologischen Geschlecht eingeschriebene Männlichkeiten wahrgenommen werden.

\section{III - Hegemoniale Männlichkeit, christlicher Masochismus und der reflexive Sadomasochismus}

Connell differenziert in seinem 1995 erstmals erschienenen Buch Masculinities unterschiedliche Männlichkeiten in hegemoniale und untergeordnete. Am unteren Ende der hierarchischen Ordnung steht der homosexuelle Mann, während er in einer späteren Veröffentlichung (Connell 1998) den transnationalen Geschäftsmann als hegemoniale Männlichkeit in Zeiten der Globalisierung betrachtet.

In Ergänzung definiert David Savran (1998) den Habitus des bürgerlich männlichen Subjekts in Einklang mit Michel Focault als auf Selbstdisziplinierung beruhend. Er sieht mit Kaja Silvermann einen moralischen Masochismus als ein diesen Habitus von Beginn an prägendes Moment. Savran leitet den für ihn hegemonialen männlichen Habitus historisch aus der Weiterentwicklung und Ausdifferenzierung dieser masochistischen Prägung her. Interessant an diesem Versuch, eine materialistisch fundierte Geschichtsschreibung mit Psychoanalyse kurzzuschließen, ist, dass zumindest zum Teil Beispiele analysiert werden, die auch für die Kulturwelt Heavy Metal wichtig sind, so z.B. der Film Easy Rider und die Biker-Kultur, die Figur des Kriegers in den Rambo-Filmen von Sylvester Stallone oder der feminisierte und sexualisierte Rockstar Jim Morrison von den Doors.

Savran (1998: 109) unterscheidet die weiß dominierte Baby BoomerGegenkultur der 1960er Jahre von der Neuen Linken inklusive der beginnen- 
den neuen Frauenbewegung und der afroamerikanischen Selbstorganisation von der Bürgerrechtsbewegung bis zu den Black Panthers. Er sieht im Habitus der Gegenkultur, die für inn von den Beatniks ausgeht und sich zu den Hippies weiterentwickelt, unterschiedliche Ausprägungen des Masochismus aufscheinen, die sich zu einem reflexiven Sadomasochismus weiterentwickeln, den er als grundlegend für die momentan hegemoniale Männlichkeit zumindest in den USA behauptet. Savran leitet diesen reflexiven Sadomasochismus aus Gedanken von Sigmund Freud und Silvermann ab:

Reflexive sadomasochism is »a condition in which the ego is ingeniously split between a sadistic (or masculinized) half and a masochistic (or feminized) half so that the subject, torturing himself, can prove himself a man « (Savran 1998: 33).

Die für meine Überlegungen zu Heavy Metal zentrale Ausprägung des Masochismus bezeichnet er wiederum nach Silvermann als christlichen Masochismus (vgl. ebd.: 108f., 126, 156ff.), der in der Gegenkultur der 1960er Jahre verstärkt zu finden ist. Dieser ist nach dem Abbild von Christus als religiösem Märtyrer modelliert. Er ordnet sein feminisiertes Sein einem höheren maskulinen Zweck unter. Die Trennung des bürgerlichen Subjekts in weibliche und männliche Anteile überschreitet damit die Grenze zwischen Phantasie und Realität, die in anderen Formen des Masochismus aufrecht erhalten wird. Damit einhergehend analysiert Savran eine Entmännlichung oder Entsexualisierung. In einer zentralen Szene des Films Easy Rider werden die beiden von Dennis Hopper und Peter Fonda gespielten Protagonisten als zumindest temporär impotent dargestellt, als Männer, die Karneval und Drogen, Mardi Gras in New Orleans und LSD realem Sex (mit bezahlten Prostituierten) vorziehen (vgl. ebd.: 108f.). Der Drogenrausch gilt Savran dabei als Möglichkeit, das feminisierte Sein auszuleben, auf das im nüchternen Zustand panisch und mit dem Wunsch nach Remaskulinisierung reagiert wird.

Der reflexive Sadomasochismus, der von Sylvester Stallones Figur John Rambo archetypisch verkörpert wird (vgl. ebd.: 197-206), verlagert diesen Kampf zwischen feminin und maskulin dagegen in das Subjekt. Durch seine permanente Selbstfolter ist Rambo durch eine reale Verweiblichung nicht mehr in seiner Männlichkeit bedroht. Die permanente Spannung, der Hass, der der internalisierten Weiblichkeit entgegengebracht wird, bricht sich auch in der Realität potentiell gewalttätig Bahn, während ein für das maskuline Selbstbild notwendiges Interesse, sich einem höheren Zweck unterzuordnen, nicht mehr existiert. 


\section{Männerbilder im Heavy Metal I - vorindustrielle und postapokalyptische Bilder}

Geht man von einer Relevanz von Safrans Annahmen aus, so muss sich diese Veränderung der Männlichkeit in den Männerbildern des Heavy Metal widerspiegeln. Heavy Metal entsteht, historisch betrachtet, aus der Asche der von Savran analysierten weißen, männlich dominierten Gegenkultur der 1960er Jahre.

Die zu verfolgende These ist, dass der männliche Habitus des Heavy Metal von Varianten des christlichen Masochismus bestimmt ist, während dessen Weiterentwicklung zu Rambo, die bei Savran beispielsweise über die phallische Aufladung des christlichen Masochismus in der Person von Jim Morrison (vgl. ebd. 129ff.) vermittelt ist, zumindest partiell oder in Ansätzen negiert wird.

Gemeinsam ist all diesen aus der Gegenkultur entwickelten Männerbildern der Bezug auf ein romantisch verklärtes präindustrielles Lebensideal. Easy Rider spielt weitgehend in ländlichen Gegenden, Rambo lebt in den Wäldern und Morrison inszeniert sich als romantischer Dichter.

Dieser idealisierte Bezug ist gleichfalls zentral für einen männlichen Habitus im Heavy Metal. So zeigt ein in Sepia designtes Pressefoto der österreichischen Dark bzw. Black Metal-Band Dornenreich die beiden Gründungsmitglieder zwischen den abgestorbenen Ästen eines Baums kauernd. Max Cavalera, der Frontmann von Soulfly, posiert mit Erde verschmiert in einer Höhle. Das Foto zeigt vor allem seinen Kopf sowie seine tätowierten Unterarme. Abbath, Sänger und Bassist der norwegischen Black Metal-Band Immortal, wirft sich vor dem Hintergrund eines norwegischen Fjordes in eine wahrscheinlich bedrohlich oder auch herrisch gemeinte Pose, während Gaahl, zum Zeitpunkt des Fotos noch Sänger der norwegischen Black MetalBand Gorgoroth, eher wachsam und wie kurz vor der Flucht in einer kargen Berglandschaft steht. Er blickt an der Kamera vorbei und beobachtet aufmerksam etwas für den Betrachter des Fotos Unsichtbares. Die beiden Letztgenannten sind schwarz gekleidet und mit Corpsepaint geschminkt.

Als Folie für diesen Bezug auf präindustrielle Lebensweisen müssen in den USA gerne Native Americans herhalten - in direkter Fortschreibung der Hippie-Identifikation - , in Großbritannien die Kelten, in Skandinavien die Wikinger und in Deutschland wahlweise die Germanen oder die literarische 
Romantik. ${ }^{4}$ Als Fußnote mag in diesem Zusammenhang die indianisch-germanische Abstammung der Figur John Rambo dienen.

In all diesen Bilder dominiert das christlich-masochistische Märtyrertum von Easy Rider noch den reflexiven Sadomasochismus von John Rambo. Die Protagonisten von Easy Rider verkörpern die Vision eines sich modernisierenden Nordamerikas und werden aufgrund dieser Bedrohung der bestehenden Ordnung von >den Anderen<, den konservativen oder beharrenden Kräften erschossen. Auch die beschriebenen, präindustriell konnotierten Heavy Metal-Bilder beinhalten eine derartige Vision, sei es die Stärkung der Gefühle in einer kalten Welt bei Dornenreich, den Kampf für die Rechte der weltweit Unterdrückten und Marginalisierten bei Max Cavalera oder der Kampf gegen die regionale Unterdrückung durch das Christentum bei Gaahl.

In einer Weiterentwicklung wird dieser romantisch verklärte männliche Habitus in der Bilderwelt des Heavy Metal gerne in Bezug gesetzt zu postindustriellen bzw. postapokalyptischen Gesellschaftsbildern. Wir sind nicht mehr die, die aktiv aussteigen wollen oder werden, sondern Opfer (der Globalisierung), die wahlweise aus dem kapitalistischen Verwertungsprozess Ausgeschlossenen oder an ihm Verzweifelnden. Zu diesem Zweck werden häufig Bilder leerer, gern verfallener Fabrikhallen und ähnlichem als Videosetting genutzt. Die von der deutschen, auf Heavy Metal spezialisierten Plattenfirma Nuclear Blast herausgegebene DVD-Reihe Monsters Of Metal beinhaltet in ihrer vierten, 2006 erschienenen Auflage 54 Werbefilme von Heavy Metal-Bands. 26 und damit fast genau die Hälfte dieser Filme spielen in verlassenen Fabrikhallen, Fabrikkellern oder ähnlichen über Rohre, Maschinen usw. als ehemals industriell genutzte Umgebungen charakterisierten Settings. In diesen postapokalyptischen Bildern treten die christlich-masochistischen Visionen zwar in den Hintergrund zugunsten eines stärker werdenden Fatalismus, gerade die strafende Instanz, die für die verfallenen Settings verantwortlich zeichnet, bleibt jedoch spürbar.

John Rambo wird ebenfalls in eine für inn feindliche Situation geworfen. Allerdings wird er nicht nur endlos gequält, sondern er quält sich auch selbst. Seine Mission, der Vietnam-Krieg, ist gescheitert und hat eine völlige innere Leere hinterlassen. Um diese zu konterkarieren, ist die Kamera besonders in Rambo II verliebt in die Darstellung des männlichen Körpers von Stallone, gleichwohl ist die Männlichkeit von Rambo nie in Gefahr, sie braucht keine strafende Instanz mehr, wie noch in Easy Rider. Gerade diese Verliebtheit in den männlichen Körper fehlt den postindustriellen Männer-

4 Beispielsweise finden sich Bezüge zu Native Americans bei Manowar, zu Kelten bei Primordial, zu Wikingern bei Amon Amarth, zum Germanentum bei Varg und zur literarischen Romantik bei Dornenreich. 
bildern im Heavy Metal. Während die Umgebung für Rambo zur bloßen Staffage für die Zelebrierung des männlichen Körpers wird, dominiert in den Heavy Metal-Videos das Setting weiterhin die Körperdarstellung.

Der reflexiv sadomasochistische Habitus des John Rambo findet sich im Heavy Metal vielmehr als weiterentwickelter Import aus der HardcoreBewegung, die ja zumindest teilweise auch als individualisierende Reaktion auf den Verlust der gesellschaftsverändernden Mission von Punk verstanden werden kann.

Eine der Bands, die für die Verschmelzung von Hardcore- und Heavy Metal-Bildsprache stehen, sind Pantera in der Besetzung mit Sänger Phil Anselmo. Im Video zu Panteras Song »Mouth Of War « (Pantera 2003), das nicht zufällig in einem undefinierten Raum spielt, streichelt die Kamera den nackten, muskulösen und tätowierten Oberkörper von Anselmo sowie seinen geschorenen Kopf, den nur ein kleiner Kinnbart von einem Skinhead oder einem Angehörigen des US-Marine-Korps unterscheidet. Die restlichen Bandmitglieder sind dagegen vollständig angezogen und präsentieren lange Haare. Von besonderem Interesse für die Kamera sind hier die in Zeitlupe eingefangenen Bilder der umherfliegenden Haare.

Diese oberflächlich homoerotische Inszenierung des Männerkörpers findet ihr heterosexuelles Pendant in Jim Morrison. Allerdings wird Sänger Phil Anselmo nicht zum Phallus im Morrisonschen Sinne, sondern zum Muskel im Sinne von Rambo. Immer enthalten ist eine mehr oder weniger latente Gewaltdrohung, die durchaus reale Gründe hat. Savran (1998: 131) schreibt, bezogen auf Morrison: »A woman may light Morrison's fire on her own risk. «

\section{Männerbilder im Heavy Metal II - Der Clown und die drogeninduzierte Unzurechnungsfähigkeit}

Black Sabbath-Sänger Ozzy Osbourne verkörpert dagegen einen männlichen Habitus, der weder die prä- oder postindustriellen noch die körperbetonten Elemente beinhaltet, die die Modernisierung des christlichen Masochismus durch den reflexiven Sadomasochismus im Heavy Metal bebildern.

Die Black Sabbath-Performance von »Paranoid« (Black Sabbath 2002) in einer 1970 ausgestrahlten belgischen TV-Show zeigt einen fröhlich lachenden Osbourne, der als einziges Bandmitglied in Samtjackett und Hemd gekleidet ist. Er wirkt eher schüchtern und verspielt und damit wenig kraftvoll, wenig körperbewusst und in gewisser Weise entsexualisiert. Er repräsentiert weder Phallus noch Muskel. Eine eher ungelenke Körperlichkeit findet sich auch in Konzertmitschnitten von »War Pigs« (1970, ebd.), und 
»Children Of The Grave« (1973, ebd.). Osbourne zeigt während »War Pigs« bei geöffnetem Cordhemd einen leichten Bauchansatz und bewegt sich in den Instrumentalpassagen wenig koordiniert um den Mikrofonständer herum, der ihm als Verankerung dient, um nicht von der Energie der Musik fortgerissen zu werden. »Children Of The Grave« wurde während eines Open Air Festivals mitgeschnitten und zeigt einen mittlerweile selbstbewussteren Osbourne mit weiterhin fröhlicher Ausstrahlung, der das Publikum zum Feiern und Mitklatschen auffordert. Er führt einen choreographierten Sprung vor, bei dem er in gehockter Position landet. Sowohl das Mitklatschen als auch und insbesondere der Sprung wirken weiterhin ungelenk. Die Kraft, die er in den beiden beschriebenen Konzertausschnitten zu verkörpern sucht, findet keinen adäquaten körperlichen Ausdruck. Im Vergleich zu den bisher beschriebenen Bildern eines männlichen Habitus von Angela Gossow bis Phil Anselmo wirkt er unbeholfen und fast tapsig. Sein Gestus erinnert im 1973 gedrehten Clip zu »Sabbath Bloody Sabbath« (ebd.) zudem an die Rolle des Klassenclowns, eine typische untergeordnete Männlichkeit. Osbourne wird während des Gesanges schelmisch grinsend und in Großaufnahme gezeigt. Seine Mundbewegungen sind dabei absichtlich nicht synchron mit der Tonspur. Ozzy Osbourne ist nicht cool und alles, was er verkörpert, ist ganz bestimmt nicht ernst gemeint.

Im Rahmen der lebensgeschichtlichen Männerforschung hat Messerschmidt (2000) in Bezug auf gewalttätige Jugendliche in den USA gezeigt, dass gerade die körperliche Konstitution entscheidend für die Position in der männlichen Peergroup ist. Eine daraus abgeleitete These ist, dass schon Ozzy Osbournes Körperlichkeit ihn nicht für eine hegemoniale Position in einer derartigen Peergroup prädestiniert.

Die Rolle des Clowns ist assoziiert mit Zirkus und Karneval und im Falle Ozzy Osbournes mit Drogen. Die beschriebene Szene aus dem Clip zu »Sabbath Bloody Sabbath« wird mit Überblendungen verschiedener Gesichter fortgeführt, die zusammen mit der wackeligen Kameraführung einen spsychedelischen oder an einen Drogenrausch erinnernden Effekt erzeugen sollen. Die Verbindung von Drogen und Karneval, die Sex vorgezogen werden, spielte bereits für Savran (1998: 108f.) eine zentrale Rolle in der Beschreibung des christlichen Masochismus in Easy Rider. Damit ist dieser auch Teil des männlichen Habitus von Ozzy Osbourne.

Die (gesellschaftsverändernde) Vision, die Teil des christlichen Masochismus ist, lässt sich mit einem Blick auf die Inhalte einiger Songtexte von Black Sabbath belegen. Zwar ist "War Pigs « ein eher fatalistisches Antikriegslied, aber bereits das ein Jahr später veröffentlichte Anti-Atombomben-Stück »Children Of The Grave« hat den Glauben an die Kraft der 
Gegenkultur wiedergefunden: »If you want a better place to live in, spread the words today / Show the world that love is still alive / You must be brave or you children of today are children of the grave, yeah! « (Black Sabbath 1971). In einigen Texten finden sich sogar explizit christliche Aussagen, so die Zeile »They should realize before they critisize that God is the only way to love (ebd.) aus dem 1971 veröffentlichten »After Forever «.

Die hippieske, protestsonghafte und durchaus auch christliche Botschaft der damals meist nicht von Osbourne stammenden Songtexte von Black Sabbath steht konträr zu ihrem bösen, okkulten bis satanischen Image. ${ }^{5}$ Heavy Metal in Gestalt von Black Sabbath hält an den gegenkulturellen Werten der 1960er Jahre fest. Die parallele Weiterentwicklung derselben über die Feminisierung des sich auf den Phallus reduzierenden Männerkörpers findet im Hard Rock von Led Zeppelin, Aerosmith und anderen statt und mündet in den Glam Metal, der mit dem Heavy Metal von Black Sabbath nicht umsonst musikalisch wenig bis nichts zu tun hat.

Glam Metal kennzeichnet sich visuell über eine äußerliche Verweiblichung des Männerkörpers mittels Kleidung, Frisur und Make-up und geht häufig mit hypermaskulinem Verhalten und übersteigerter Frauenfeindlichkeit einher (vgl. Strauss 2002). In der wissenschaftlichen Rezeption (vgl. Denski/Sholle 1992) werden die Genderstereotype verstärkenden Elemente des Glam Metal gegenüber den Genderstereotypen angreifenden Elementen betont. Beide fehlen bei Osbourne weiterhin, ähnlich wie die bereits beschriebene Reduktion der Körperlichkeit auf Phallus oder Muskel und der Bezug auf prä- oder postindustrielle Settings. Allerdings muss angemerkt werden, dass sich Osbourne zu Beginn seiner Solokarriere in den 1980er Jahren visuell an den damals in den USA kommerziell dominanten Glam Metal annähert und beispielsweise plötzlich lockiges Haupthaar zeigt.

Wichtiger erscheint mir jedoch eine andere, direkter von Osbourne ausgehende Akzentverschiebung im männlichen Habitus, die sich in der Weiterentwicklung der Rolle des Clowns in Richtung (drogeninduzierte) Unzurechnungsfähigkeit manifestiert. Damit einhergehend wird die gegenkulturelle Aufladung des Drogengebrauchs gleichzeitig negiert und fortgeschrieben. In der Negierung mutieren Drogen zum unterhaltsamen Selbstzweck, der die Nähe zum gesamtgesellschaftlich akzeptierten Feierabendbier sucht. Im Rahmen des männlichen Habitus im Heavy Metal führt dies zur Akzeptanz

5 Die Fortschreibung des satanischen Images bringt Osbourne zum einen in den 1980er Jahren eine Anklage wegen Beihilfe zum Selbstmord ein, die jeder Grundlage entbehrt (vgl. Walser 1993: 147-151), und wirft zum anderen ein Schlaglicht auf die weitgehende Bedeutungslosigkeit von Songtexten im Heavy Metal. 
von dementsprechend gezeichneten Männerkörpern, also zum Beispiel des Bierbauches.

Die Fortschreibung der gegenkulturellen Aufladung des Drogengebrauchs geschieht im exzessiven Drogenmissbrauch, dessen Ziel jedoch keine Bewusstseinserweiterung sondern eine Bewusstseinsauslöschung ist und in diesem Ziel auch mit Thesen Timothy Learys zum LSD-Gebrauch korreliert (vgl. Savran 1998: 126). Für Savran (ebd.) manifestiert sich im Wettbewerb des >Wer verträgt mehr von welcher Droge auch immer auch die für Rambo typische Selbstfolter, die der Männlichkeit keinen Abbruch tut.

Der Versuch, den christlichen Masochismus der 1960er Jahre im Männerbild des Heavy Metals zu konservieren, erhält so erste Risse und wird offen für Modernisierungen wie die weiter oben bei Pantera beschriebene Körperlichkeit, die bei diesen gerade auch trotz exzessivem Drogenmissbrauch und drogeninduzierter Unzurechnungsfähigkeit erhalten werden muss. ${ }^{6}$

Exzessiver Drogenmissbrauch jeglicher Art begleitet die gesamte Karriere Ozzy Osbournes, allerdings gelangt er - bezogen auf das Image - erst in den 1980er Jahren parallel zu Glam Metal zu voller Blüte. Während der Star in den 1970er Jahren unpässlich war, aber schon wieder arbeitsfähig werden wird, ist die Unzurechnungsfähigkeit jetzt Teil des zu vermarktenden Images. Erster Höhepunkt ist 1982 das Abbeißen des Kopfes einer echten toten Fledermaus, die Osbourne fälschlicherweise für ein Plastikimitat hält. Osbourne stilisiert sich in der Folge als verrückt. Spätestens in der Einleitung zum 1993 als Video veröffentlichten Konzertmitschnitt Live \& Loud (Osbourne 1993) wird bewusst mit der (drogeninduzierten) Unzurechnungsfähigkeit geworben. Man suggeriert real nicht existente Bilder des so genannten sbat biting v von 1982, zieht gleichzeitig mit den zu sehenden schwarz-weiß Aufnahmen eines unzurechnungsfähigen Osbourne eine Verbindungslinie in die 1970er und thematisiert auch heterosexuellen Sex über eine kurz ins Bild rückende barbusige Konzertbesucherin. Die Begrüßung der Fans zu Beginn des Konzerts endet dementsprechend mit den Worten: »I'm Ozzy and I'm crazy.«

6 Sänger Phil Anselmo war aufgrund chronischer Rückenschmerzen drogenabhängig und 1996 nach einer Heroinüberdosis bereits klinisch tot. Die auf Home Videos von Pantera (vgl. Pantera 1997) dokumentierten, vor allem von Alkohol getriebenen Späße der restlichen Bandmitglieder sollen als Inspiration für die auf MTV gezeigte Show Jackass gedient haben (vgl. Bittner 2009: 22). 


\section{Männerbilder im Heavy Metal III - Psychopathisches}

Osbournes immer noch im Karneval verhaftete Unzurechnungsfähigkeit ist mittlerweile perfektionierten Images des nach Savran (1998: 156f.) ebenfalls im christlichen Masochismus verwurzelten Psychopathischen gewichen. Irrenanstalten und Zwangsjacken sind im zeitgenössischen Heavy Metal beispielsweise der Apokalyptischen Reiter oder der Legion of the Damned ein gern genutztes Setting für Videoclips und Fotos und verbinden sich mit den beschriebenen postapokalyptischen Bildern. Sie verkörpern wiederum die Existenz einer strafenden Macht, deren Präsenz den christlichen Masochismus vom reflexiven Sadomasochismus unterscheidet. Stärkere Verbindungen zu Rambos Selbstfolter finden sich im ebenfalls verbreiteten Gebrauch von masochistischem Sexspielzeug als karnevalesk entsexualisiertes Entertainment, das sich sowohl bei Rammstein, den Apokalyptischen Reitern, der Black Metal-Band Urgehal als auch bei zahllosen anderen finden lässt. Ein Pressefoto von Urgehal kennzeichnet beispielsweise den männlichen Psychopath, der durch einen der Gitarristen der Band verkörpert wird, mit einer mit überdimensionalen Nieten bewehrten Ledermaske und einem ledernen Brustgeschirr. Er ist blutbespritzt und hält eine Machete in der linken Hand; der Fotohintergrund zeigt ebenfalls blutbespritzte Kacheln. Dies kann als wenig subtile Bebilderung der von Savran für den reflexiven Sadomasochismus beschriebenen Verlagerung des Kampfes zwischen feminin und maskulin in das Subjekt gelesen werden. Noch deutlicher werden Urgehal bei den Plattencovern zu Through Thick Fog Till Death (2003) und Demonrape (2005), die die Auslöschung bzw. Beherrschung des Femininen durch die eben beschriebene männliche Figur explizit bebildern. Dagegen nutzen Rammstein und Die Apokalyptischen Reiter masochistische Ikonographie besonders gerne als Teile der Bühnenkleidung ihrer Keyboarder und konnotieren damit das Spiel von Tasteninstrumenten als potentiell weiblich, bedrohlich und > unmetallisch<. ${ }^{7}$

7 Für eine genauere Beleuchtung des mit der Verwendung sadomasochistischen Sexspielzeugs verbundenen Verhältnisses des männlichen Habitus im Heavy Metal zu schwuler S/M-Sexualität, die ebenfalls bereits in den 1970er Jahren beginnen muss, fehlt hier leider der Platz. Stattdessen ein Textausschnitt aus dem 1977 erschienenen Song »Raw Deal« von Judas Priest (Sin After Sin):

»I made the Spike Bar nine o'clock on a Saturday / All eyes hit me as I walked into the door / Them steel and leather guys were fooling with the denim dudes / A couple cops playin' rough stuff, New York, fire island [...] Sex like a hurricane, it ravaged and it shattered / I was barely holding on to this flying body 


\section{Fazit}

Die mit Bourdieu charakterisierte männliche Herrschaft wird von der Kulturwelt Heavy Metal nicht in Frage gestellt. Alle beschriebenen Männerbilder zeigen eine unterschiedlich starke Annäherung des für Heavy Metal als grundlegend behaupteten christlich-masochistischen Habitus an den mit Pierre Bourdieu (2005) und David Savran (1998) als hegemonial definierten reflexiv sadomasochistischen Habitus des weißen Mannes. Allerdings erfährt diese Bewegung in der Kulturwelt sowohl Zustimmung als auch Ablehnung und führt zu sehr unterschiedlichen Männerbildern, für die die beschriebenen psychopathischen, prä- und postindustriellen Bild- und Videosettings, die feminisierten und gleichzeitig hypermaskulinen Bildwelten des Glam Metal sowie der muskulöse und durchtrainierte Hardcore-Einfluss beispielhaft stehen sollen. Als besonders resistent gegen eine Modernisierung ihres in der Gegenkultur der 1960er Jahre verankerten männlichen Habitus erweist sich Heavy Metal in der Folge von Ozzy Osbourne und Black Sabbath. Osbourne versucht zwar dem reflexiven Sadomasochismus des hegemonialen männlichen Habitus relativ konsequent zu entkommen, nähert sich aber über die zum Imageträger erkorene drogeninduzierte Unzurechnungsfähigkeit doch wieder an.

Den modernisierenden Effekt dieses Wunsches nach Kontrollverlust beschreibt der Soziologe Keith Kahn-Harris (2007: 27-49) als Teil einer Suche nach Transgression, die er als zentrales Element der Kulturwelt Heavy Metal ansieht. Er thematisiert Transgression als eine Dialektik von Kontrollwunsch und Kontrollverlust, die er auf einer Haltung der »reflexive anti-reflexivity« (ebd.: 145) beruhend sieht, also des Es-zwar-besser-Wissens, aber Es-trotzdem-lieber-nicht-wissen-Wollens. Kahn-Harris Bezug auf Mikhail Bakhtins (1984) Ideen zum mittelalterlichen Karneval schafft eine direkte Verbindungslinie zu Osbournes ebenfalls im Karneval geerdeter Unzurechnungsfähigkeit und Savrans (1998: 108f.) Ausführungen zu Easy Rider. Die dunkle Macht, vor der Ozzy Osbournes Protagonist im Aufsatztitel flüchtet, kann damit - metaphorisch gesprochen - als die momentan hegemoniale Männlichkeit beschreiben werden, deren Anziehungskraft für die Kulturwelt trotzdem unbestritten bleibt.

symphony / I guess I dream in pictures, not in colors / The true free expression I demand is human rights - right / I hate my life, I am an actor." 


\section{Literatur}

Bakhtin, Mikhail (1984). Rabelais and His World. Bloomington: Indiana University Press.

Bayton, Mavis (1997). »Women and the Electric Guitar."In: Sexing the Groove. Popular Music and Gender. Hg. v. Sheila Whiteley. London: Routledge. S. 37-49.

Bittner, Ronny (2009). » Hell Yeah!<. Das Leben und Schaffen des Dimebag Darrell.« In: Rock Hard 271, S. 20-25.

Bourdieu, Pierre (1998). Praktische Vernunft. Zur Theorie des Handelns. Frankfurt/M.: Suhrkamp.

Bourdieu, Pierre (2005). Die männliche Herrschaft. Frankfurt/M.: Suhrkamp.

Brecht, Bertold (1964). Schriften zum Theater (7 Bd.). Hg. v. Werner Hecht. Berlin, Weimar: Aufbau.

Christie, Ian (2003). Sound of the Beast - the Complete Headbanging History of Heavy Metal. New York: Harper Collins.

Connell, R.W. [Raewyn] (1995). Masculinities. Berkeley, Los Angeles: University of California Press.

Connell, R.W. (1998). »Masculinities and Globalization.«In: Men and Masculinities $1 / 1$, S. 3-23.

Denski, Stan / Sholle, David (1992). »Metal Men and Glamour Boys. Gender Performance in Heavy Metal. «In: Men, Masculinity and the Media. Hg. v. Steve Craig. Newbury Park, London, New Delhi: Sage, S. 41-60.

Engler, Steffani (2008). »Habitus und sozialer Raum: Zur Nutzung der Konzepte Pierre Bourdieus in der Frauen- und Geschlechterforschung." In: Handbuch Frauen- und Geschlechterforschung. Theorie, Methoden, Empirie. Hg. v. Ruth Becker und Beate Kortendiek. Wiesbaden: VS Verlag für Sozialwissenschaften (2. erw. und aktualisierte Aufl.), S. 250-261.

Fast, Susan (2009). » Girls Rock Your Boys! • The Continuing Non-History of Women in Rock Music." In: History/Herstory. Alternative Musikgeschichten. Hg. v. Annette Kreutziger-Herr und Katrin Losleben (= Musik-Kultur-Gender Band 5). Köln, Weimar, Wien: Böhlau, S. 154-172.

Früchtl, Josef / Zimmermann, Jörg (2001). "Ästhetik der Inszenierung. Dimensionen eines künstlerischen, kulturellen und gesellschaftlichen Phänomens.« In: Ästhetik der Inszenierung. Dimensionen eines künstlerischen, kulturellen und gesellschaftlichen Phänomens. Hg. v. Josef Früchtl und Jörg Zimmermann. Frankfurt/M.: Suhrkamp, S. 9-48.

Gaar, Gillian G. (1994). Rebellinnen. Die Geschichte der Frauen in der Rockmusik. Hamburg: Argument.

Kahn-Harris, Keith (2007). Extreme Metal. Music and Culture on the Edge. Oxford, New York: Berg.

Messerschmidt, James (2000). Nine Lives. Adolescent Masculinities, the Body, and Violence. Boulder, CO: Westview Press.

Savran, David (1998). Taking It Like a Man. White Masculinity, Masochism, and Contemporary American Culture. Princeton, NJ: Princeton University Press.

Strauss, Neil und Mötley Crüe (2002). The Dirt. Planegg: Hannibal.

Walser, Robert (1993). Running with the Devil. Power, Gender and Madness in Heavy Metal Music. Hanover, London: Wesleyan University Press.

Weinstein, Deena (1991). Heavy Metal - A Cultural Sociology. New York: Lexington. 
Weinstein, Deena (2009). »The Empowering Masculinity of British Heavy Metal.« In: Heavy Metal Music in Britain. Hg. v. Gerd Bayer. Farnham: Ashgate, S. 17-32.

Willems, Herbert (2009). "Zur Einführung: Medientheatralität und Medientheatralisierung. «In: Theatralisierungen der Gesellschaft 2: Medientheatralität und Medientheatralisierung. Hg. v. Herbert Willems. Wiesbaden: VS Verlag, S. 13-38.

Zimmermann, Marc (2003). »Gestus. «In: Wörterbuch der Theaterpädagogik. Hg. v. Gerd Koch und Marianne Streisand. Uckerland: Schibri. Online unter: http://www.ash-berlin.eu/theaterpaed-wb/index.phtml?action=anzeigen\&id=22 (Zugriff am 22.2.2010).

\section{Diskographie}

Black Sabbath (1970). »Black Sabbath«. Auf: Black Sabbath. Vertigo 832702-2.

Black Sabbath (1971). »After Forever«, »Children Of The Grave«. Auf: Master Of Reality. Vertigo 6360050.

Judas Priest (1977). »Raw Deal«. Auf: Sin After Sin. CBS 32005.

Urgehal (2003). Through Thick Fog Till Death. Agonia Records ARlp 005.

Urgehal (2005). Demonrape. Agonia Records ARep 011.

\section{Filmographie}

Black Sabbath (2002). »Paranoid«, »War Pigs«, »Children Of The Grave«, »Sabbath Bloody Sabbath«. In: The Black Sabbath Story Volume One. DVD. Sanctuary Visual Entertainment SVEM0051.

Dunn, Sam / McFayden, Scott (2006). Metal - A Headbanger's Journey. DVD. München: Constantin.

Easy Rider (1969). Regie: Dennis Hopper. Columbia Tristar Home Video [Neuauflage 1999].

Osbourne, Ozzy (1993). Live \& Loud. Regie: Jeb Brien. Columbia [Neuauflage 2003].

Pantera (1997). Pantera 3. Watch It Go. Warner Home Vision 8536 40195-3.

Pantera (2003). »Mouth of War«. Auf: Reinventing Hell. The Best Of. CD + DVD. Elektra Records, Rhino Records 8122-73729-2.

Rambo. First Blood (1983). Regie: Ted Kotcheff. Kinowelt 500160 [Neuauflage 2000].

Rambo II. Der Auftrag (1985). Regie. George Pan Cosmatos. Kinowelt [Neuauflage 2006].

V.A. (2006). Monsters Of Metal. The Ultimate Metal Compilation DVD Vol. 4. Nuclear Blast 2736115082.

V.A. (2009). Maximum Metal 147. DVD-Beilage zur Metal Hammer-Ausgabe vom Januar 2010. München: Axel Springer Young Mediahouse. 


\begin{abstract}
Based on David Savran's thesis, that the contemporary hegemonic male habitus is based on reflexive sadomasochism and is itself an advancement of a Christian masochism rooted in 1960s counterculture, male heavy metal images are analysed to detect at least traces of this change of the male habitus in heavy metal culture. Most of the analysed images - preindustrial, postapocalyptic, clown, psychopath are still rooted in Christian masochism. But heavy metal is also attracted by reflexive sadomasochism to experience the pleasures of transgression central to heavy metal culture.
\end{abstract}

